\title{
A Rare Complication of Patent Ductus Arteriosus Ligation: Inadvertent Ligation of the Left Pulmonary Artery
}

\author{
Semiha Terlemez, ${ }^{1}$ Onur Işık, ${ }^{2}$ Suzan Şahin, ${ }^{3}$ Abdullah Barış Akcan, ${ }^{3}$ Münevver Kaynak Türkmen ${ }^{3}$ \\ ${ }^{1}$ Adnan Menderes University Medıcıne Faculty, Pediatrıc Cardıology Department, Aydın, Turkey; ${ }^{2}$ Tepecik Training and \\ Research Hospital, Cardiovascular Surgery Department, İzmir, Turkey; ${ }^{3}$ Adnan Menderes University Medıcıne Faculty, \\ Neonatology department, Aydın, Turkey
}

\section{ABSTRACT}

Background: The inadvertent ligation of the left pulmonary artery (LPA) is a rarely seen surgical complication that has been presented in the literature in a limited number of cases after patent ductus arteriosus (PDA) ligation surgery.

Case Report: A PDA closure operation was performed on our patient, a 28-week-old preterm. In the postoperative follow-up, we identified on echocardiography taken on the same postoperative day that the ductus space was still present. On CT angiography, we determined that not only was the ductus space still continuing, but, in addition, ligation of the LPA had been performed inadvertently. An LPA reconstruction operation was performed on the patient 46 days after the first operation. However, owing to severe tissue damage in LPA, LPA reperfusion did not occur in the postoperative period.

Conclusion: Although inadvertent ligation of the left pulmonary artery during PDA ligation surgery is rarely seen in patients who have undergone closure surgery, this complication should be kept in mind in the postoperative follow-up period. Patient findings such as physical examination, lung angiography and postoperative echocardiography should be assessed with this in mind.

\section{INTRODUCTION}

Patent ductus arteriosus (PDA) ligation is one of the congenital heart surgery operations regarded as relatively easy. Nonetheless, although rare, there can be complications. These include involvement of the descending aorta (Ao) or inadvertent ligation of the left pulmonary artery (LPA), which are complications with considerably high mortality and morbidity rates. Inadvertent LPA ligation during PDA ligation is still reported in the literature [Panagopoulos 1971; Pontius 1981; Jaffe 1986; Oezel 1986; da Costa e Silva 2010; Tefera 2015] The reoperation of these patients did not always yield successful results. Some patients died [da Costa e Silva 2010] and LPA and left lung hypoplasia developed in others [Jaffe

Received November 1, 2016; received in revised form fune 8, 2017; accepted fune 25, 2017.

Correspondence: Semiba Terlemez, Adnan Menderes University Medicine Faculty Pediatric Cardiology Department, Aydin, Turkey 09100; 90-532-721-7689; (email:semihaterlemez@yahoo.com).
1986]. In some patients, however, satisfactory results were obtained [Tefera 2015]. Factors affecting the success of reconstructive surgery in these patients are not clear. Here we present a patient born as preterm and who underwent an inadvertent LPA ligation in the course of surgical ductus closure.

\section{CASE REPORT}

Our patient was born at 28 weeks gestation and weighed 1080 grams by cesarean section to a mother with gestational diabetes. In the follow-up, surfactant was given and the patient was put on mechanical ventilator support. The patient was still in need of respiratory support and it was recommended to have medical ductus closure after hemodynamics showed a significant PDA $(3.5 \mathrm{~mm})$ on echocardiography. Medical ductus closure treatment was given with 2 doses of ibuprofen; the first while the patient was 6 days old and the second at 10 days old. Nonetheless, the PDA gap was still present. Therefore, supportive care including digoxin and diuretics was initiated for the patient. When the patient was 30 days old, dilation of the left cardiac cavities was seen to have developed. PDA ligation was decided upon, and when the infant was 33 days old, the PDA ligation surgery was carried out. At the second hour of postoperative evaluation of the patient, a murmur below the left sternum was noticed on physical examination. When it was evaluated with echocardiography, the ductus gap was observed to be there. However, no suspicious view was seen in echocardiography with $2 \mathrm{D}$ in the distal sections of the left pulmonary artery, and no pathology was seen in doppler flow. Reduction in the left parenchymal volume attracted attention in the preoperative and postoperative images of the chest xray (Figure 1). Due to the
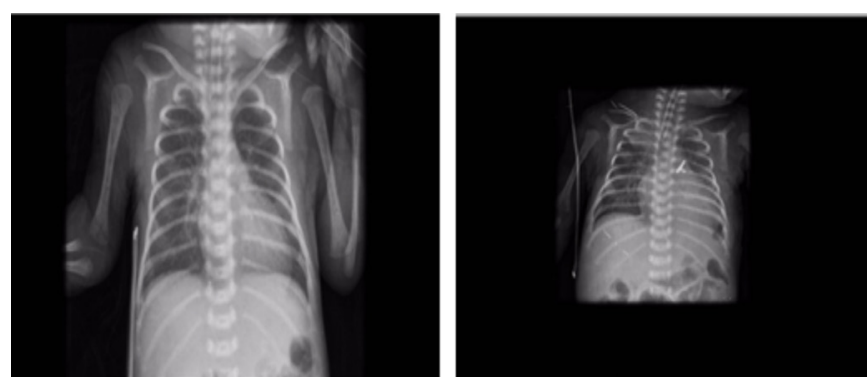

Figures $1 \mathrm{a}$ and $1 \mathrm{~b}$. Chest xray before and after the ductus operation. 

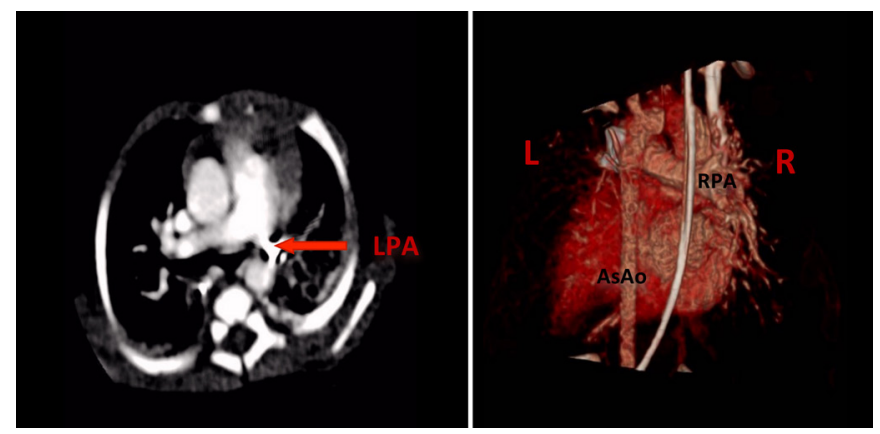

Figures $2 \mathrm{a}$ and $2 \mathrm{~b}$. Transverse section CT angiography image and threedimensional CT angiography images. Distal segments after clip are not seen in LPA. LPA, Left Pulmonary artery; RPA, right pulmonary artery; AsAo, ascending aorta.

continuation of ductus flow, a CT angiography was taken. It was seen in the angiography that a left pulmonary artery ligation had been performed (Figure 2).

The overall condition of the patient deteriorated including sepsis after a short time from the first operation, then generally recovered after the patient was followed-up for a long time with inotrope support and appropriate antibiotic treatment. The second operation was performed on the patient 46 days following the first operation. LPA reconstruction and PDA ligation were undertaken through a median sternotomy incision. Only the clips were removed without an LPA incision during reconstruction. PDA ligation was carried out. Extensive tissue damage was observed in the LPA up to the hilus section in intraoperative evaluation. Anticoagulant treatment (clexan) was initiated to contribute to LPA reperfusion by taking into account the vascular endothelium impairment in the postoperative period. It was seen that there was no reperfusion in the LPA in CT angiography taken after 2 months following the reconstruction operation. During the follow-up of the patient, respiratory distress increased and widespread atelectasis developed in the left lung. The patient died about 6 months after reconstructive surgery.

\section{DISCUSSION}

LPA ligation is a rare complication of ductus closure operations [Panagopoulos 1971; Jaffe 1986]. Clinical findings, time to diagnosis, and reconstructive surgery results of patients presented in the literature show differences in outcomes. In the present study, we had difficulty in diagnosing the patient although we carried out echocardiography on the same postoperative day. PDA flow was observed on the LPA distal segment in classical sections of echocardiography. Since a PDA gap was still present, it brought to mind this complication, and LPA ligation was then determined by CT angiography. When other postoperative lung angiographies were examined with this awareness, it caught our attention that there was a reduction in left lung parenchyma volume and drift to the left of the heart. We did not identify a hyperlucent image in left lung that was identified in previous case reports [da Costa e Silva 2010].

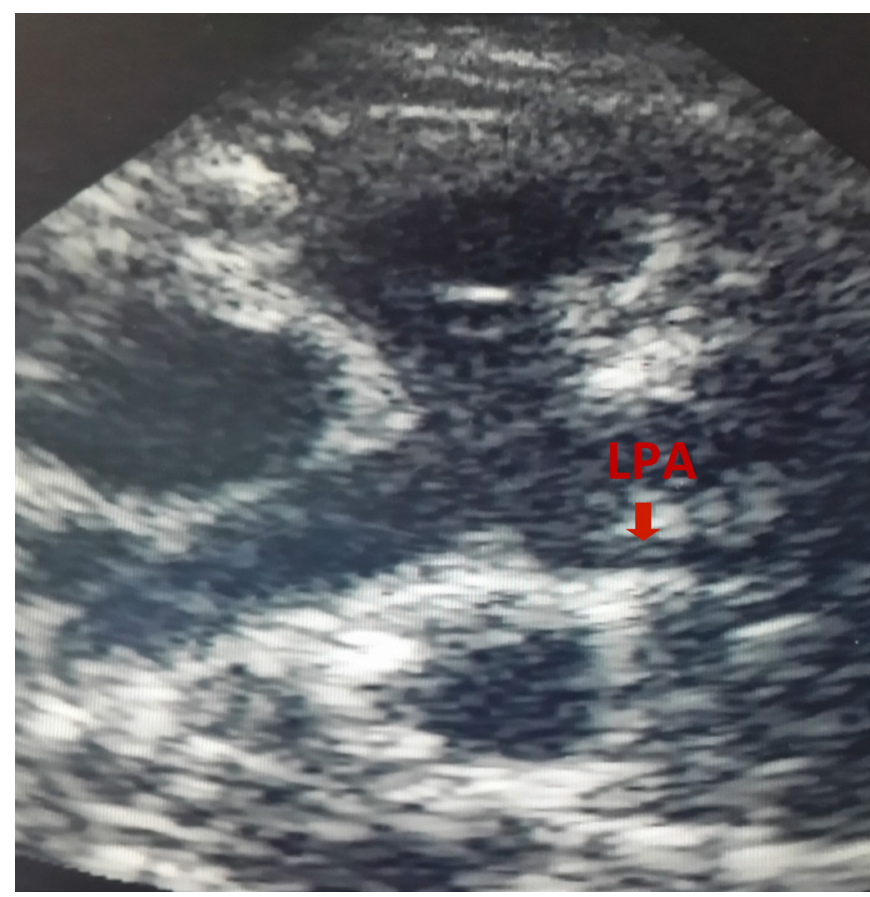

Figure 3. LPA image in parasternal short axis section echocardiography after reconstructive surgery.

Patients whose reperfusion had been restored after reconstructive surgery have been presented in the literature [Tefera 2015]. We were not able to ensure reperfusion in our patient following reconstructive surgery. The success of the surgery could be related to many factors, such as tissue damage developed in LPA, the technique of PDA ligation operation (the number of used clips, etc.), and the duration until reconstructive surgery. The distal LPA looked occluded and severely damaged following reconstruction in our patient (Figure 3). The patient died about 6 months after reconstructive surgery.

There is no experienced team in congenital heart surgery in our hospital. We think that the inexperience of the surgeon may have played a role in this complication. The patient's ductus ligation was done at our hospital. However, reconstructive surgery was performed at another center with more experience. We do not know the answer to the question if reperfusion would have been restored had the reconstruction operation been performed earlier. We did the patient's reconstruction surgery 46 days after the first operation. Because we diagnosed the LPA ligation two weeks after the operation, we expected the patient to recover and the sepsis to improve. Even though we noticed that something was wrong in the post-operative evaluations, the LPA ligation did not come to mind in the early period.

As a result, although the PDA ligation seems to be a simple operation, it is better if it is done by experienced surgeons. It is necessary to be aware of this possible complication when evaluating patients in the early postoperative period after PDA ligation in order to get the diagnosis early and to treat the patient with the least damage. When assessing patients from this point of view, it is imperative to refer to auscultation 
findings, hints in postoperative lung angiographies, and above all, postoperative echocardiography images.

\section{REFERENCES}

da Costa e Silva EJ, de Albuquerque SC. 2010. Left pulmonary artery ligation. Pediatr Radiol 40:S86.

Jaffe RB, Orsmond GS, Veasy LG. 1986. Inadvertent ligation of the left pulmonary artery. Radiology 161:355-357.

Orzel JA, Monaco MP. 1986. Inadvertent ligation of the left pulmonary artery instead of patent ductus arteriosus. Noninvasive diagnosis by pulmonary perfusion imaging. Clin Nucl Med 11:629-631.

Panagopoulos PHG, Tatooles CJ, Aberdeen E, et al. 1971. Patent ductus arteriosus in infants and children: a review of 936 operations (1946-69). Thorax 26:137-144.

Pontius RG, Danielson GK, Noonan JA, Judson JP. 1981. Illusions leading to surgical closure of the distal left pulmonary artery instead of the ductus arteriosus. J Thorac Cardiovasc Surg 82:107-113.

Tefera E, Bermudez-Cañete R, van Doorn C. 2015. Inadvertent ligation of the left pulmonary artery during intended ductal ligation. BMC Res Notes 8:511. 